\section{Innate stimuli integrator}

The phosphatase MKP-1 is known as a negative regulator of innate immune responses induced by Toll-like receptor signaling in macrophages. In Immunity, Chi and colleagues show that induction of MKP-1 by pathogen-recognition receptors in DCs controls the reciprocal development of T helper type 1 cells and IL-17producing helper T cells. MKP-1-deficient DCs induce a greater frequency of IL-17+ T cells and a lower frequency of interferon- $\gamma$ positive T cells in vitro and in vivo without affecting the survival or proliferation of T cells or the expression of type 2 cytokines. The authors show that MKP-1 inhibits activation of the kinase p38 and subsequently modulates the expression of IL-12 and IL-6 in DCs. In addition, MKP-1 inhibits TGF- $\beta$ expression and the generation of inducible regulatory T cells. Thus, MKP-1 acts in DCs to integrate innate stimuli to regulate the differentiation of T helper type 1 cells, IL-17-producing helper T cells and inducible regulatory T cells. IV Immunity 35, 45-58 (2011)

\section{KIR selection of HIV}

Natural killer (NK) cell activity is regulated by target-cell recognition via polymorphic killer immunoglobulin-like receptors (KIRs). In Nature, Alter et al. show that HIV-infected people select for variant viruses with point mutations that enhance the interaction of inhibitory KIRs with infected $\mathrm{CD}^{+} \mathrm{T}$ cells. The HIV-1 genome has 22 sequence polymorphisms that can be associated with a specific inhibitory human KIR in HLA-C $1^{+}$people. For example, HIV-1 variants with viral protein $\mathrm{U}(\mathrm{Vpu})$ with methionine and histidine at positions 71 and 74, respectively, are significantly more prevalent in KIR2DL2 ${ }^{+}$patients, in contrast to HIV-1 variants with arginine and leucine at those positions, which are associated with KIR2DL2 ${ }^{-}$patients. Cells infected with the former variant are more resistant to killing mediated by KIR2DL $2^{+} \mathrm{NK}$ cells but retain sensitivity to KIR2DL2 ${ }^{-}$NK cells. Thus, NK cells can contribute to viral control, but this selective pressure leads to the outgrowth of viruses that can evade NK cell-mediated killing. It remains unclear how viral mutations lead to differences in KIR-HLA recognition.

$L A D$

Nature (4 Aug 2011) doi:10.1038/nature10237

\section{Link to NF-kB}

CIN85 is an adaptor known for its interaction with the E3 ubiquitin ligase $\mathrm{Cbl}$; it has been proposed to function in endocytosis of membrane receptors. In the Journal of Experimental Medicine, Kometani et al. report that CIN85 links the B cell antigen receptor to activation of the canonical NF- $\mathrm{kB}$ pathway. B cellspecific deletion of CIN85 does not effect the induction of $\mathrm{T}$ cell-dependent antibody responses or germinal center formation. However, T cell-independent responses are almost entirely lost because of the defective survival and proliferation of mutant $B$ cells after stimulation with antibodies to immunoglobulin $M$. CIN85 deficiency results in less activation of the kinase IKK complex, whereas activation of noncanonical NF- $\mathrm{kB}$ downstream of lipopolysaccharide, the costimulator CD40 and the activator BAFF remains intact. In addition, the development of B-1 cells is substantially impaired in CIN85-deficient mice, which further supports the idea that CIN85 is involved in signal transduction mediated via the $B$ cell antigen receptor.

J. Exp. Med. 208, 1447-1457 (2011)

\section{Keys to $\beta$-selection}

The differentiation of thymocytes into the $\alpha \beta$ or $\gamma \delta$ lineage occurs at the $\beta$-selection checkpoint. Differences in the signal strength of the $\gamma \delta$ T cell antigen receptor (TCR) or precursor TCR are thought to govern this lineage fate, albeit in a ligand-independent way. In Science Signaling, Pennington and colleagues rule out previous models that suggested that surface receptor oligomerization controls this selection process. Thymocytes deficient in the precursor to TCR $\alpha$ (preT $\alpha)$ reconstituted with a natural splice variant encoding preT $\alpha$ that lacks a charged immunoglobulin-like domain that mediates receptor oligomerization are able to drive cells to proliferate and differentiate to the double-positive stage. Similarly, transfection of RAG2-deficient thymocytes with $\gamma \delta$ TCR constructs that lack extracellular domains, which thereby prevents receptor oligomerization, can still promote double-positive differentiation of $\gamma \delta$ thymocytes. The formation of signaling-competent $\gamma \delta$ TCR or TCR $\beta-$ pre-T $\alpha$ in double-negative thymocytes seems to be the minimum requirement for cell progression. Thus, the authors argue that signal-strength differences are probably due to regulation of receptor abundance. $L A D$ Sci. Signal. (19 July 2011) doi:10.1126/scisignal.2001765

\section{Putting a KAP on infection}

The integration of viral cDNA into the host genome is a key step in the life cycle of retroviruses. In Cell Host and Microbe, Cereseto and colleagues identify a host restriction factor that interferes with the integration of human immunodeficiency virus type 1 (HIV-1). Retroviral integration requires a viral integrase whose activity is enhanced by host acetylases. A screen of host proteins that interact with acetylated viral integrase identifies KAP1 (TRIM28) of the transmembrane adaptor TRIM family. KAP1 inhibits integration of and infection with HIV-1, and it seems to do this by forming a complex with the deacetylase HDAC1 and binding to integrase targets. Once bound to integrase, the KAP1-HDAC1 complex deacetylates it and thereby decreases its activity and ability to facilitate viral integration. The function of KAP1 seems to be wholly dependent on deacetylation, as it is ineffective in the absence of HDAC1, even if KAP1 is overexpressed. This study extends the family of factors able to restrict viral infection and identifies an important step in the viral life cycle at which they may act.

\title{
Lung fibroblasts call the shots
}

Important lung pathologies such as chronic obstructive pulmonary disease (COPD) are caused by the coordinated action of a variety of cellular interactions and secreted factors such as transforming growth factor- $\beta$ (TGF- $\beta$ ). In the Journal of Clinical Investigation, Nishimura and co-workers pinpoint lung fibroblasts as key participants in lung inflammation and COPD. In a model of COPD induced by interleukin $1 \beta$ (IL-1 $\beta$ ), they observe that IL-1 $\beta$ results in higher expression of the integrin $\alpha_{\mathrm{v}} \beta_{8}$ on the surface of lung interstitial fibroblasts. This integrin is required for cleavage and activation of TGF- $\beta$. Active TGF- $\beta$ then binds to its receptor on fibroblasts in an autocrine or paracrine way, which results in release of the chemokines CCL2 and CCL20. Both of these chemokines can recruit dendritic cells (DCs) to lung-draining lymph nodes, where they prime T cell responses. Although not formally demonstrated, lung epithelial damage, such as by smoke particulates, may trigger IL-1 $\beta$ release and act as an initiating factor. Fibroblasts therefore seem to have a central role in COPD and potentially other forms of pathological lung inflammation.

J. Clin. Invest. 121, 2863-2875 (2011) 